\title{
Editorial
}

\section{How're We Doin'? Preventing Occupational Infections With Blood-Borne Pathogens in Healthcare}

\author{
David K. Henderson, MD
}

Ed Koch was the 105th Mayor of New York City and served in this capacity for three terms from 1978 to 1989. During his time as mayor, he was recognized for restoring financial stability and accountability to the "Big Apple," but was nearly equally as famous for walking the streets of New York and asking of random constituents, "How'm I doin'?" In many, if not most, respects, I believe his "walking-thetalk" behavior should be a paradigm for the healthcare industry in the 21 st century.

The arrival of acquired immunodeficiency syndrome (AIDS) and human immunodeficiency virus (HIV) infection into the healthcare milieu in the early 1980 s provided perhaps the single greatest thrust to improve the safety of healthcare practices regarding occupational risks for exposure to, and infection with, blood-borne pathogens. In this issue of Infection Control and Hospital Epidemiology, four articles address issues relating to healthcare workers' risks for, and prevention of, occupational infections with bloodborne pathogens. ${ }^{1-4}$ As we approach the 25 th anniversary of the identification of AIDS and HIV infection, we may all gain from taking a careful, thorough, and thoughtful look at how we're doin' regarding preventing exposures to and infections with blood-borne pathogens in the healthcare setting-ultimately, with an eye toward the development of new, and even more effective, prevention strategies.

The creation by the Centers for Disease Control of the so-called universal precautions guidelines ${ }^{5}$ (now broadened to encompass the concepts associated with standard precautions) ${ }^{6}$ represented the first giant step toward reducing risks for occupational infections of healthcare workers with blood-borne pathogens. These guidelines focused principally on primary prevention (ie, preventing occupational exposures to blood and thereby preventing infection with all blood-borne pathogens) and emphasized-because testing can never provide $100 \%$ accuracy - that blood from all patients should be assumed to represent occupational risk. This concept, which arrived on the healthcare scene only with the implementation of the universal precautions guidelines in 1987, should have long since permeated the healthcare workplace, beginning in the late $1940 \mathrm{~s}$, when the occupational risks for hepatitis B infection were initially identified and characterized. ${ }^{\text {? }}$

Since the publication of the universal precautions guidelines, we have learned a great deal about primary prevention of occupational exposures (Table). In my view, thoughtful adherence to these precautions likely has contributed more to the reduction of occupational risk than any other single intervention. These guidelines effectively put the healthcare worker in the "driver's seat" regarding risk management. If, in the healthcare worker's view, a specific procedure is associated with a risk for getting blood on his or her hands, the healthcare worker should elect to wear gloves. If a risk for spattering or splattering is present, the healthcare worker could decide to protect his or her mucous membranes. That it took until the late 1980 s for these guidelines to appear is problematic. Nonetheless, the major message of these guidelines-that blood equals risk in the healthcare environment-is both precisely correct and entirely reasonable.

Prospective evaluation of procedures and work practices associated with recurring risk is another area rife with potential for reducing occupational risk. In my institution, a multidisciplinary team effectively mines all of our exposure data, looking for common circumstances of exposure. In instances in which a specific procedure is identified as being associated with recurring risk, we propose and test modifications to the procedure in an attempt to prevent exposures. Use of this approach during the past 15 years 


\section{TABLE}

Primary Prevention Strategies to Reduce the Risk for OCCUPATIONAI EXPOSURE TO, AND INFECTION With, BloODBORNe PATHOGENS

Thoughtful use of universal or standard precautions

Educational interventions

Retraining existing staff about the presence and magnitude of occupational risks

Modification of medical-nursing-dental school curricula to include emphasis on risks from blood-borne pathogens

Periodic reinforcement and updating of training related to these infections

Modification of procedures and work practices that are intrinsically risky or that are associated with frequent exposures

Use of safer devices (ie, engineered controls or technologic advances) Immunization

has resulted in a substantial reduction in the frequency of parenteral exposures self-reported to the Occupational Medicine Service of the National Institutes of Health (Figure). Whereas we can take solace in the fact that the 3 in 1,000 risk for HIV infection associated with a single discrete parenteral exposure to blood from an HIV-infected patient ${ }^{8}$ is a relatively small risk, it is not an occupational risk that one would ever elect to take on a regular or repetitive basis.

Similarly, prospective evaluation of the risks associated with the use of specific devices is another area that has provided great fodder for prevention. The use of creative, engineering controls-new and safer devices-has also contributed to substantial reduction of risk. ${ }^{9,10}$ Again, in my institution, our multidisciplinary team processes all of our exposure data, focusing on both the related devices and the circumstances of exposure, to try to identify specific factors associated with institutional risk. When a particular device is incriminated as being associated with increased risk, the multidisciplinary team scans the current economic environment for potentially safer devices. When a putatively safer device is identified, the team carefully evaluates it in our environment.

In this issue of Infection Control and Hospital Epidemiology, Sohn et al. use a similar approach to assess the efficacy of implementing a "safer-needle system" in their institution. ${ }^{1}$ In this study, the simultaneous implementation of several "safer" devices resulted in a reduction of self-reported parenteral exposures by a remarkable $58 \%$. Whereas these results are encouraging, their study does have limitations and substantial room for additional improvement still exists. The exposure data in their study are self-reported. Whereas the authors reassure us that, "There were no secular changes or changes in systems, policies, procedures, or other factors that would have affected the mean or median number of procedures performed .....", no data are provided to support this assertion, and experience would suggest that many aspects of healthcare change (both abruptly and substantially) over

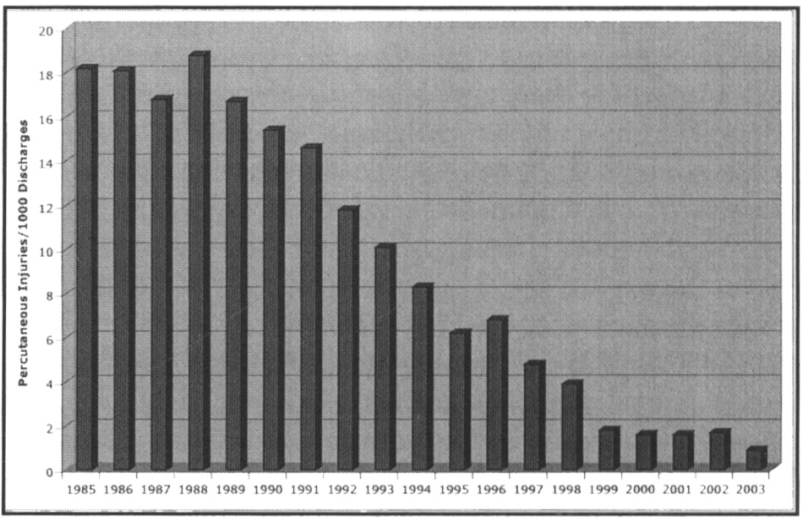

FIGURE. Parenteral injuries reported by staff to the Occupational Medicine Service per 1,000 patient discharges at the Clinical Center, National Institutes of Health, 1985 to 1993.

time. Any study using historical controls suffers from this weakness; however, I would note that the options for study design were limited, given the legal requirements for implementing the Needlestick Safety and Prevention Act of 2000 (Public Law No. 106-430, November 6, 2000).

Two of the major potential confounders of any study of parenteral exposures are concern about underreporting and the potential for reporting bias. In a second article from the same institution, Sohn et al. provide evidence that the study itself and the implementation of new safety devices in their institution did not introduce reporting bias in their study. ${ }^{2}$ The manner in which the data for the components of the study were collected makes firm or direct comparisons impossible; nonetheless, the data that the authors could generate provide support for their conclusion that the results from their postintervention study were likely not confounded by the introduction of the new devices or the conduct of the study itself.

These two studies from Memorial Sloan-Kettering Cancer Center did generate some highly disturbing data. First, a substantial number of parenteral or percutaneous exposures are continuing to occur and be reported formally in this institution (ie, $40 \%$ of a large number of exposures is still a relatively large number of exposures). Second, many of the reported postintervention injuries resulted from exposure to one of the new safety devices that had not been activated or used appropriately. Finally, the employees who self-report the largest numbers of parenteral exposures through the institution's anonymous questionnaire process are also the employees least likely to report any exposures formally, thereby, presumably, rendering them unavailable for optimal postexposure management of their exposures. If historical precedent is a good predictor, presumably, many of these individuals are our surgical colleagues, and are at the highest risk for occupational exposures and infections. Each of these problems is longstanding and all desperately need creative solutions and approaches.

In the third article related to occupational exposures 
to blood-borne pathogens in this issue of Infection Control and Hospital Epidemiology, Vaughn et al. surveyed both healthcare providers and infection control professionals primarily from smaller rural hospitals in Iowa in an attempt to assess compliance with needle safety recommendations, as well as to evaluate institutional support for exposure prevention activities. ${ }^{3}$ These investigators used a response of "never recapping" as a surrogate marker for healthcare workers' adherence to safe needle handling practices. They also used data from the American Hospital Association's (AHA) annual guide to hospitals to create institutionally based variables. Their statewide data are robust enough to be able to provide the investigators with a sense of intrainstitution variability of responses, as well as the power to assess differences among institutions in Iowa.

The inclusion of the survey data from the AHA presents a reasonably unique approach for their analysis, providing at least some insight into the relative importance of leadership and infection control infrastructure to successful implementation of needle safety programs. From the study description, I cannot discern how closely the healthcare worker survey and the AHA survey are tied in time. If they are separated by several years, the analysis may be less relevant. I also am troubled by the manner in which the investigators have constructed the dichotomous variable for needle recapping. The "never" answer is undoubtedly correct from the "purist" (or perhaps Occupational Safety and Health Administration) perspective; nonetheless, I suspect that most, if not all, of us have been in clinical circumstances in which recapping was the best available of a few bad choices. I would have been more comfortable if this variable had been "recap less than 5\%" (or perhaps even "recap less than $2 \%$ "), or, alternatively, if the investigators had compared the analysis using $100 \%$ and $95 \% / 98 \%$ and demonstrated no difference. I am concerned that intelligent, stellar, and responsible employees might never answer "never." I believe that the fact that the intensive care unit, emergency department, and operating room staffs answered "never" less frequently could reflect the urgency of their work, could reflect their inattention to infection control details, could provide indirect evidence supporting my concerns, or could represent a combination of these possibilities.

Despite these limitations, the findings from this study are simultaneously encouraging and discouraging. The fact that committed leadership, adequate infection control infrastructure, increased availability of standard precautions training, and adequate availability of personal protective equipment were all associated with decreased recapping behaviors should be reassuring to all hospital epidemiology professionals. Conversely, even if one accepts the definition of "adherent" given by Vaughn et al., I would emphasize that fewer than half of the healthcare workers analyzed in the study (47\%) were adjudged to be adherent-an almost appalling fraction for the year 2004.

The fourth article dealing with occupational exposures to blood-borne pathogens in this issue of Infection Control and Hospital Epidemiology provides an estimate of the annual number of parenteral exposures sustained by hospital-based healthcare personnel for the years 1997 and 1998 in the United States. ${ }^{4}$ Panlilio et al. constructed a model to assess the number of needlestick and other parenteral exposures that occurred in U.S. hospitals in 1997 and 1998, based on reporting rates in the National Surveillance System for Health Care Workers (NaSH) and data from the 45 hospitals in the University of Virginia's Exposure Prevention Information Network consortium that report and share occupational exposure data annually. The results of their analysis-made more powerful by using data from the annual NaSH survey to correct for underreporting and then weighting the data by numbers of admissions per institution-are clearly sobering. These investigators calculated that nearly 400,000 occupational parenteral exposures to blood likely occurred among U.S. healthcare workers in each year of the 2-year study period.

To return to former New York Mayor Ed Koch, what do these four articles, as well as the past 20 years' experience, teach us about how we're doin' managing occupational risks for infections with blood-borne pathogens in the healthcare setting? From my perspective, I would characterize both these newer articles and our extensive experience in the universal or standard precautions era as presenting truly "mixed" results.

Healthcare professionals in general and hospital epidemiology personnel in particular have worked hard to measure the risks associated with managing patients infected with blood-borne pathogens in the healthcare setting and have taken steps to reduce these risks. Successful riskreduction strategies have included, where possible, vaccination; the use of newly designed, appropriate infection control precautions; the modification of intrinsically risky procedures to make them safer; and the use of technologic advances (ie, "safer" devices) to reduce occupational risks for exposures. Many of these risk-reduction strategies have been remarkably successful and have resulted in substantial reductions in injuries at institutions around the United States that are tracking these exposures (Figure).

For example, successful implementation of vaccination programs for hepatitis B virus (HBV) infection in the United States has been associated with substantial reductions in occupational HBV infections among U.S. healthcare workers as well as reductions in the annual numbers of healthcare worker deaths from HBV infection in the United States. Additionally, during the past 15 years, new strategies have been developed for postexposure management of occupational exposures to HIV, including the use of postexposure prophylaxis with antiretrovirals. ${ }^{11,12}$ The combination of primary prevention activities and effective postexposure management strategies has been at least temporally associated with a clear reduction of occupational HIV infections reported to the Centers for Disease Control and Prevention, with no occupational infections among healthcare workers reported to the Centers for Disease Control and Prevention since 1999. ${ }^{13}$

Similarly, new strategies are being developed for the postexposure management of occupational exposures to 
hepatitis $\mathrm{C}^{14}$ and other emerging blood-borne pathogens. Thus, substantial progress has been made in reducing occupational risks during the past 20 years. Conversely, all of these new articles demonstrate that parenteral exposures to blood still occur at alarming rates in many U.S. hospitals. We still have only a rudimentary understanding of the factors that contribute to healthcare workers' on-thejob behaviors, ${ }^{15}$ and, specifically, we have an extremely limited understanding of what influences us, as healthcare providers, to take the unnecessary risks that we often do on an ongoing basis. The entire area of healthcare worker behavioral health is almost desperately in need of detailed investigation.

In my view, for healthcare to make it to the next level in terms of effective exposure prevention and management, I believe the healthcare industry will have to make it substantially easier for healthcare workers routinely to "do the right thing." To accomplish this complex feat-in addition to vaccines, effective infection control procedures, safer procedures, and safer devices-we almost certainly will need to have a clearer understanding of the factors that influence practitioner behavior, as well as the human factors that will make "doing the right thing" easier.

Several issues relating to the postexposure management of occupational exposures with blood-borne pathogens require further elucidation, including developing a clearer understanding of the early events in the biology and pathogenesis of occupational infection; developing strategies to improve the clinical care of individuals who sustain exposures (ie, making certain that an exposure has actually occurred, eliminating the overuse of chemoprophylaxis ${ }^{16}$; ensuring access to clinicians who have expertise in administering antiretrovirals; developing strategies to increase adherence with prophylaxis regimens; developing strategies to deal with antiviral resistance; and developing safe and effective strategies for managing exposures among pregnant healthcare workers.

In an era of healthcare that is increasingly being characterized by burdensome forms of all kinds, performance measurement, balanced scorecards; performance improvement metrics, and mandatory outcomes reporting, all of us might be well served by intense "Kochian introspection" regarding how we're doin' in understanding and motivating our colleagues to reduce occupational risks for infections with blood-borne pathogens.

\section{REFERENCES}

1. Sohn S, Eagan J, Sepkowitz KA, Zuccotti G. Effect of implementing safety-engineered devices on percutaneous injury epidemiology. Infect Control Hosp Epidemiol 2004;25:536-542.

2. Sohn S, Eagan J, Sepkowitz KA. Safety-engineered device implementation: does it introduce bias in percutaneous injury reporting? Infect Control Hosp Epidemiol 2004;25:543-547.

3. Vaughn TE, McCoy KD, Beekmann SE, Woolson RF, Torner JC, Doebbeling BN. Factors promoting consistent adherence to safe needle precautions among hospital workers. Infect Control Hosp Epidemiol 2004:25:548-555.

4. Panlilio AL, Orelien JG, Srivastava PU, et al. Estimate of the annual number of percutaneous injuries among hospital-based healthcare personnel in the United States, 1997-1998. Infect Control Hosp Epidemiol 2004;25:556-562.

5. Centers for Disease Control. Recommendations for prevention of HIV transmission in health-care settings. MMWR 1987;36(suppl 2):1S-18S.

6. Garner JS. Guideline for isolation precautions in hospitals. Infect Control Hosp Epidemiol 1996;17:53-80.

7. Leibowitz S, Greenwald L, Cohen I, et al. Serum hepatitis in a blood bank worker. JAMA 1949;140:1331-1333.

8. Henderson DK, Fahey BJ, Willy ME, Schmitt JM, Carey K, Koziol DE. Risk for occupational transmission of human immunodeficiency virus type 1 (HIV-1) associated with clinical exposures: a prospective evaluation. Ann Intern Med 1990;113:740-746.

9. Jagger J, Hunt EH, Brand-Elnaggar J, Pearson RD. Rates of needle-stick injury caused by various devices in a university hospital. $N$ Engl J Med 1988;319:284-288.

10. Jagger J. Preventing HIV transmission in healthcare workers with safer needle devices. Presented at the Sixth International Conference on AIDS; June 20-23, 1990; San Francisco, CA.

11. Henderson DK. HTV postexposure prophylaxis in the 21st century. Emerg Infect Dis 2001;7:254-258.

12. Henderson DK, Gerberding JL. Human immunodeficiency virus in the healthcare setting. In: Mandell GL, Dolin R, Bennett JE, eds. Principles and Practice of Infectious Diseases, ed. 6. Philadelphia: ChurchillLivingstone; 2004.

13. Do AN, Ciesielski CA, Metler RP, Hammett TA, Li J, Fleming PL. Occupationally acquired human immunodeficiency virus (HIV) infection: national case surveillance data during 20 years of the HIV epidemic in the United States. Infect Control Hosp Epidemiol 2003;24:86-96.

14. Henderson DK. Managing occupational risks for hepatitis C transmission in the healthcare setting. Clin Microbiol Rev 2003;16:546-568.

15. Rabaud C, Zanea A, Mur JM, et al. Occupational exposure to blood: search for a relation between personality and behavior. Infect Control Hosp Epidemiol 2000;21:564-574.

16. Bangsberg D, Goldschmidt $\mathrm{RH}$. Postexposure prophylaxis for occupational exposure to HIV. JAMA 1999;282:1623-1624. 